\title{
Comparison of electron width models for fast line profile calculations
}

\author{
CARlos A. IGLesias \\ Lawrence Livermore National Laboratories \\ P.O. Box 808, Livermore, CA 94550, USA
}

\begin{abstract}
The first non-vanishing term in the perturbation expansion of the electron contribution to the line width, commonly used in spectral line broadening by plasmas, was previously expressed in terms of the thermally averaged bremsstrahlung Gaunt factor. The approximations in the derivation, however, suggest that the result is uncertain. The electron width formula is tested with the hydrogen Balmer series and found suspect. Calculations for the He II Lyman series also display similar difficulties. The limitation of this electron width formulation is traced to the absence of an explicit strong collision cutoff beyond which the second-order theory is invalid.
\end{abstract}

Key words: Stark broadening; electron line widths

E-mail: iglesias1@1lnl.gov (C. A. Iglesias)

(C) 2015. This manuscript version is made available under the Elsevier user license 


\section{Introduction}

Spectral lines provide valuable information [1] and Stark-broadening models have been developed $[2,3,4]$ to characterize plasmas [5,6,7]. These models share approximations such as quasi-static ions and impact electrons [1]. Due to the large number of calculations often necessary to analyze spectra, these efforts employ fast algorithms to compute the width contribution from perturbing electrons. One approach expresses the first non-vanishing term in the perturbation expansion of the electron width as the thermally averaged bremsstrahlung Gaunt factor [8]. This formula was successfully tested for transitions involving atomic levels with low principal quantum numbers $(\mathrm{PQN})$ [8]. Contrary to other approaches [2,3,9], the Gaunt factor expression [8] does not include an explicit strong collision cutoff beyond which perturbation theory becomes invalid. Consequently there remained uncertainty whether the formula extends to spectral lines associated with higher PQN's.

Unfortunately, plasma experiments are challenging and data often contain large uncertainties making it difficult to discriminate among models. One notable exception is the high-precision measurements of the hydrogen Balmer series [10]. The purpose here is to compare two electron width models $[2,8]$ for fast line profile calculations at the conditions of the measured spectra. This requires an extension of the O'Brien-Hooper formalism [8] to neutral radiators readily accomplished from their main results. The comparisons show that the connection to bremsstrahlung used in line profile calculations $[4,7,8,11,12]$ is suspect. A second test for the He II Lyman series yields similar conclusions. Possible consequences in the analysis of recent opacity experiments [6] are entertained. The electron width models are also applied to isolated lines in Li-like systems and compared with measurements [13].

\section{Electron widths}

The line shape function for an ion emitting or absorbing (the radiator) a photon of energy $\hbar \omega$ while immersed in a plasma is given in the 'standard' Stark-broadening theory by [1,9]

$$
I(\quad)=\underset{o}{d F W}(F) J(, F)
$$

where $W(F)$ is the ion electric microfield probability distribution function and (neglecting lower state broadening to simplify notation)

$$
J(\omega, F)=-\pi^{-1} \operatorname{Re} \operatorname{Tr}_{a}\left\{\vec{d} \cdot[i \Delta \omega-i \vec{d} \cdot \vec{F} / \hbar+\phi(\Delta \omega)]^{-1} \rho \vec{d}\right\}
$$


Here, $\operatorname{Tr}_{a}$ denotes a trace over the internal states of the radiator, $\vec{d}$ is the radiator dipole operator, describe the radiator internal state populations, and the matrix elements of the detuning frequency from line center are given by

$$
=\quad+
$$

with $\hbar \omega_{\alpha}$ the energy of the radiator internal state .

The description of electron broadening in often assumes weak collisions of duration much shorter than the internal radiator state lifetimes involved in the radiative transition [1]. Hence, collisions are treated in perturbation theory retaining only the first non-vanishing term in the electron-radiator interaction simplified to the dipole approximation. The resulting second-order electron width is then given by

$$
\phi(\Delta \omega)=-\frac{\vec{d} \cdot \vec{d}}{3 \hbar} \int_{o}^{\infty} d t e^{i \Delta \omega t} \operatorname{Tr}_{e}\left\{\vec{F}_{e} \cdot e^{-i H_{e} t / \hbar} \rho_{e} \vec{F}_{e} e^{i H_{e} t / \hbar}\right\}
$$

where $\operatorname{Tr}_{e},{ }_{e}$, and $H_{e}$ denote a trace, density matrix, and Hamiltonian for an electron gas, respectively, and $\vec{F}_{e}$ is the electric field produced by the electron gas at the radiator. In the line formulations of interest here, the electron width is rewritten in the form

$$
\phi(\Delta \omega)=-\frac{4 \pi n_{e} e^{2}}{3 \hbar} \sqrt{\frac{2 m}{\pi T}} \vec{d} \cdot \vec{d} G(\Delta \omega)
$$

where $m, n_{e}$, and $T$, are the electron mass, number density, and temperature (energy units), respectively, and the $G$-function is related to the space and time dependent charge-density fluctuations in an electron gas [14]. Two approaches for computing the $G$-function [2,8] that are commonly used in experimental plasma diagnostics are compared in the present work.

\subsection{Lee model}

The second-order width can be written in terms of an integral over the momentum transfer in the electron-radiator collision [2,14]. Assuming $\hbar \Delta \omega \ll T$ yields

$$
\phi(\Delta \omega) \approx \frac{2 e^{2} T}{3 \pi \hbar \Delta \omega} \vec{r}_{a} \cdot \vec{r}_{a} \int_{o}^{k_{m}} d k k^{2} \operatorname{Im} \varepsilon^{-1}(k, \Delta \omega)
$$

leading to

$$
G(\quad)=\int_{o}^{k} \frac{d k}{k} \exp \left\{\frac{1}{2 k_{e}^{2} \frac{2}{p}}\right\} \frac{1}{|(, k)|^{2}}
$$


where $e^{\text {and }}{ }_{p}$ are the electron Debye length and plasma frequency,

$$
\begin{aligned}
& { }_{e}^{2}=\frac{T}{4 n_{e} e^{2}} \\
& { }_{p}^{2}=\frac{4 n_{e} e^{2}}{m}
\end{aligned}
$$

$e$ is the elementary unit of electric charge, and $(, k)$ is the dielectric function for a homogeneous electron gas. Thus, the latter neglects the net radiator charge effects on the perturbing electrons. Nevertheless, it is expected to be valid for charged radiators [9].

The cutoff $k_{m}$, which limits the momentum transfer in a collision and avoids the divergence of the integral, can be estimated by considering the partial wave expansion of the full electronradiator interaction $[15,16]$, which contains the spherical Bessel function $j_{\ell}\left(k r_{a}\right)$ [17] with $r_{a}$ the radial position of the bound electron. In most cases of interest $k r_{a}<<1$ and the lowest order nonvanishing term yields the dipole approximation. Thus, a reasonable upper limit is given by $k$ values that are cutoff by fast oscillations in $j_{\ell}\left(k r_{a}\right)$ [15],

$$
\frac{\hbar^{2} k_{m}^{2}}{2 m} \approx E_{j}
$$

where $E_{j}$ is the binding energy of the radiator $\mathrm{j}^{\text {th }}$ level. For hydrogenic systems,

$$
k_{m}^{2} a_{o}^{2} \quad \frac{Z^{2}}{n^{2}}
$$

with $Z e$ the nuclear charge, $n$ the level PQN, and $a_{o}$ the Bohr radius. Further approximations replace the dielectric function by its $\rightarrow 0$ and $\infty$ limits to get [2]:

$$
\begin{gathered}
G_{o}=\frac{1}{2} \ln 1+k_{m}^{2} e_{e}^{2} \frac{k_{m}^{2} e^{2}}{1+k_{m}^{2} e^{2}} \\
G_{\infty}(\quad)=\frac{1}{2} E_{1}\left(\frac{1}{2 k_{m}^{2} e_{e}^{2}} \frac{2}{2}\right)
\end{gathered}
$$

with $E_{1}(z)$ the exponential integral [17]. Finally, Lee takes [2]

$$
G_{L}(\quad)={ }_{n}+\operatorname{Min}\left[G_{o}, G_{\infty}(\quad)\right]
$$


where neglected strong collisions are accounted by ${ }_{2}=1.5,{ }_{3}=1.0,{ }_{4}=0.75, \quad{ }_{5}=0.5$ and ${ }_{n}=0.4$ for $n>5[9,18]$. For large detuning,

$$
G_{L}(\rightarrow \infty)={ }_{n}+k_{m}^{2} \underset{e}{2} \frac{{ }_{p}^{2}}{2} \exp \left(\frac{1}{2 k_{m}^{2} e_{e}^{2}} \frac{2}{p}\right)
$$

Thus, a correction is necessary to reproduce the quasi-static limit [2]. The Griem et al. [9] and Calisti et al. [3,18] models are similar to Lee's [2] and need not be considered separately.

The subroutines to compute Stark profiles of one- and two- electron systems with $G_{L}$ were incorporated into widely used codes for designing and analyzing experiments $[19,20]$. In addition, the subroutines were generously made available for other applications [21].

\subsection{O'Brien-Hooper model}

An alternative expression for the $G$-function is given by the thermal average [8]

$$
G_{O H}\left(\quad ; Z_{\text {net }}\right)=\frac{\pi}{\sqrt{3}} \int_{o}^{\infty} \frac{d E}{T} e^{E / T} g\left(E, \quad ; Z_{\text {net }}\right)
$$

where $g\left(E, \quad ; Z_{\text {net }}\right)$ is the bremsstrahlung Gaunt factor for absorption of a photon with energy $\hbar \Delta \omega$ by an electron with initial energy $E$ scattering in the Coulomb potential produced by the net radiator charge, $Z_{n e t} e$. It can be expressed in terms of the radial integrals $[8,22,23,24]$

$$
\int_{o}^{\infty} d r \psi_{\ell}(r, E) \psi_{\ell \pm 1}(r, E+\hbar \Delta \omega)
$$

for partial wave $\ell$ and boundary conditions for the perturbing electron wave function

$$
\begin{aligned}
& \ell(r \rightarrow 0, E) \propto r^{\ell+1} \\
& \ell(r \rightarrow \infty, E) \propto \frac{\sin \left(q r+{ }_{\ell}\right)}{q r}
\end{aligned}
$$

with wave vector $\hbar^{2} q^{2} / 2 m=E$ and $\quad$ the phase shift.

In the case of neutral radiators $\quad \ell(r, E)=j_{\ell}(q r)$, the spherical Bessel function in the partial wave expansion of plane waves. Thus, the Gaunt factor reduces to the Born approximation [24] with thermal average [25]

$$
\begin{aligned}
G\left(\Delta \omega ; Z_{\text {net }}=0\right) & =G_{O H}^{B o r n}(\Delta \omega) \\
& =e^{\hbar \omega / 2 T} K_{o}(\hbar \Delta \omega / 2 T)
\end{aligned}
$$


where $K_{o}(z)$ is the modified Bessel function of the second kind [17]. For large detuning

$$
G_{O H}^{\text {Born }}(\quad \rightarrow \infty) \propto \quad 1 / 2
$$

reproducing the Holtsmark quasi-static limit [2]. The $G$-function expression in Eq. (2.2.1) describes ideal electrons interacting with the net radiator charge, however, electron-screening effects can be approximated by $G_{O H}\left(={ }_{p}\right)$ for $\leq{ }_{p}$ [26,27]; thus, avoiding the wellknown infrared divergence [28]. There is no correction for strong collisions in the O'BrienHooper formulation.

\section{Results}

In this Section the hydrogen Balmer spectrum is computed with $G_{O H}$ and $G_{L}$, which requires the extension of the O'Brien-Hooper formalism to neutral radiators above. The line widths near line center for the He II Lyman series are compared. The $\mathrm{Mg} \mathrm{He \gamma}$ line profiles, used to characterize plasmas in opacity measurements [6], provide an example of systematic errors from these electron width formulas. Finally, the widths of the $3 \mathrm{~s}-3 \mathrm{p}$ isolated lines of Li-like systems, which present well-known challenges for Stark-broadening theory [13], are considered.

\subsection{H Balmer series}

The Balmer emission spectrum was measured with high precision in a wall-stabilized arc [10] providing a test for Stark broadening theories. A spectrum calculation using the line shape codes from Lee [2] was in good agreement with measurements [21] and is reproduced in Fig. 1. Level populations were computed [29] as well as the radiative recombination into the hydrogen $L$-shell and emission from the $H+e$ system to compare with the experimental spectrum.

The present work does not compare directly with the experimental spectra. Instead, it assumes the profiles with $G_{L}$, as shown in Fig. 1, are in good agreement with the measurements. Thus, only the emission bound-bound spectrum is calculated with standard Stark-broadening theory [1] plus Doppler broadening using level populations in thermal equilibrium. The electric microfield distribution in the quasi-static ion approximation uses the APEX model for neutral radiators [30] while the electrons are treated with either $G_{L}$ or $G_{O H}^{B o r n}$ explicitly retaining the frequency dependence of the electron broadening. All dipole allowed couplings between initial levels with PQN $n=3$ through $n=20$ are included. The final states are restricted to the $n=2$ levels with coupling between $2 \mathrm{~s}$ and $2 \mathrm{p}$ but without any coupling to the initial levels except for 
the radiative transitions, which include all dipole allowed lines from $n=3,4 \ldots 20$ to $n=2$. The calculations neglect interference terms [1]. A Hessenberg decomposition algorithm is used to accelerate the integration over the microfield distribution at each detuning frequency [31].

The area normalized Balmer emission bound-bound spectra at conditions comparable to the experiments [10] are displayed in Fig. 2. The plots show significant discrepancies with $G_{O H}^{B o r n}$ producing broader lines and a faster approach to the Inglis-Teller limit [32]. Thus, these comparisons together with the agreement between calculations using $G_{L}$ and the measurements suggest $G_{O H}^{B o r n}$ overestimates the electron widths.

The ratios of the electron widths $G_{O H}^{B o r n} / G_{L}$ for the two electron densities in Fig. 2 computed near line center $\left(={ }_{p}\right)$ are given in Table 1. The table shows, consistent with the spectra in Fig. 2, a discrepancy between the electron broadening models increasing with PQN. Note that $G_{O H}^{B o r n}$ remains fixed independent of the transition; it is $G_{L}$ that decreases with increasing upper level PQN as a result of the decreasing momentum transfer cutoff in Eq. (2.1.5).

It follows from the $\rightarrow \infty$ limit of the $G$-functions that the discrepancy increases in the line wings. The ratios $G_{O H}^{B o r n} / G_{L}$ for the $H \beta$ line at the conditions in Fig. 2 are plotted in Fig. 3 as a function of detuning frequency. The curves in the figure terminate at the estimated value of the Weisskopf frequency, which for a hydrogenic system with nuclear charge $Z e$ is given by [2]

$$
\hbar \omega_{k f} \approx \frac{3 Z \hbar}{4 n^{2} a_{o}} \sqrt{\frac{3 T}{m}}
$$

and approximates the onset of quasi-static electrons. Both formulas have the $\quad{ }^{1 / 2}$ behavior for $>{ }_{k f}\left(\right.$ imposed on $G_{L}$ ) but the approach, as seen in Fig. 3, is significantly different.

\subsection{Modified O'Brien-Hooper model}

As mentioned above, $G_{O H}^{B o r n}$ does not contain a strong collision cutoff. It is possible to introduce a minimum impact parameter in the electron-radiator collision following the arguments for $k_{m}$, which are equivalent to excluding perturbing electrons from penetrating the radiator. For the sum over partial waves, then [24] 


$$
\begin{array}{ll}
\ell_{\min } & q R \\
& \frac{3 n^{2}}{2 Z} \sqrt{\frac{2 a_{o} E}{e^{2}}}
\end{array}
$$

where $E$ and $\hbar q$ are the initial scattering electron energy and momentum, respectively, $R$ estimates the size of the bound electron orbit, and the second line assumes a hydrogenic radiator with nuclear charge Ze and level with PQN $n$. The modified O'Brien-Hooper $G$-function is then given by limiting the sum over partial waves to $\ell \ell_{\min }$. That is,

$$
\tilde{G}_{O H}^{B o r n}(\omega)=\frac{\pi}{2} \int_{o}^{\infty} \frac{d E}{T} e^{-E / T} \sqrt{\frac{E+\hbar \omega}{E}} \sum_{\ell \geq \ell_{\min }} \ell \Gamma^{2}(\ell) X^{\ell}\left\{A_{\ell}^{2}(X)+B_{\ell}^{2}(X)\right\}
$$

where

$$
\begin{aligned}
& \left(\ell+\frac{1}{2}\right) A_{\ell}(z)={ }_{2} F_{1} \quad \frac{1}{2}, \ell, \ell+\frac{1}{2} ; z \div \\
& \left(\ell+\frac{3}{2}\right) B_{\ell}(z)=\frac{\sqrt{z}}{2}{ }_{2} F_{1} \frac{1}{2}, \ell, \ell+\frac{3}{2} ; z \div
\end{aligned}
$$

with ${ }_{2} F_{1}(a, b, c ; z)$ and $(z)$ the hypergeometric and Gamma functions [17], and

$$
X=\frac{E}{E+\hbar \omega}
$$

obtained using

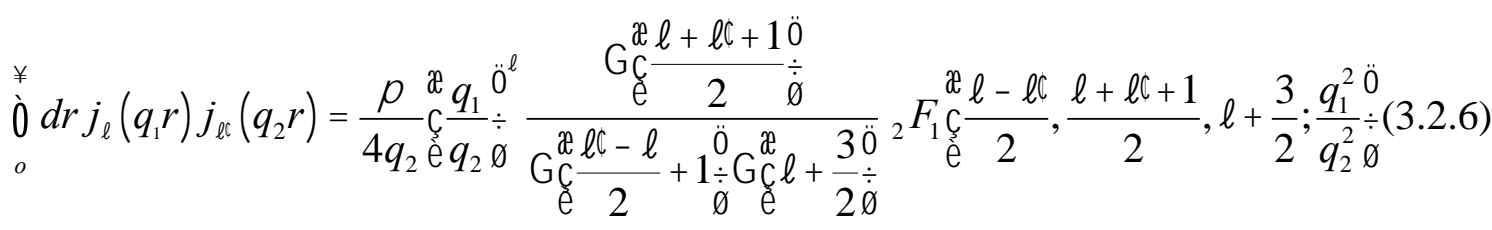

Due to the large number of partial waves required to obtain accurate results, it is often more efficient to compute the sum for $\ell<\ell_{\min }$ and subtract the average from $G_{O H}^{B o r n}$ in Eq. (2.2.4).

The ratios $\tilde{G}_{O H}^{B o r n} / G_{L}$ evaluated at $\quad={ }_{p}$ are given in Table 1 displaying improved agreement (values closer to unity) with $G_{L}$. Also displayed in the table are values for $\ell_{\min }$ calculated at $E=T$ showing that significant contributions from the sum over partial waves are excluded in the modified $G$-function for high PQN's. The frequency dependent ratio $\tilde{G}_{O H}^{B o r n} / G_{L}$ is plotted in Fig. 3 for the $H \beta$ line showing improved agreement with $G_{L}$. 
The O'Brien-Hooper formulation does not contain any correction for missing strong collisions [8]. If strong collisions are added to $G_{O H}^{B o r n}$ it increases the disagreement with $G_{L}$ for all PQN's in Table 1. Adding the strong collision correction to $\tilde{G}_{O H}^{B o r n}$ yields

$$
1.2 \leq\left(\sigma_{n}+\tilde{G}_{O H}^{B o r n}\right) / G_{L} \leq 1.8
$$

with the larger ratios occurring for the larger PQN's. That is, for larger PQN the strong collision correction ${ }_{n}$ is considerable but unfortunately uncertain.

\subsection{He II Lyman series}

The O'Brien-Hooper electron widths were first applied to the He II Lyman- $\alpha$ line [8] and later to more ionized charged radiators $[4,7,11,12]$. The ratio $G_{O H} / G_{L}$ computed at $\quad={ }_{p}$ for the He II Lyman series at three electron densities are provided in Table 2. The comparisons show $25 \%$ agreement between the Lee and O'Brien-Hooper widths for Lyman- $\alpha$, where the latter were shown [8] to be in excellent agreement with classical paths calculations [33] at the middle density in Table 2. Subsequent measurements of the He II Lyman- $\alpha$ line [34] reported experimental line widths in agreement with the unified theory [35] as well as the classical paths calculations [33]; thus, implying agreement with the O'Brien-Hooper model. As in Table 1, the ratio $G_{O H} / G_{L}$ in Table 2 increases for higher PQN's as well as with increasing electron density. In this case, adding a strong collision correction to $G_{O H}$ would negatively impact the agreement with experiments as well as increase the ratio $G_{O H} / G_{L}$ for the larger PQN's.

\section{$3.4 \mathrm{Mg} \mathrm{He \gamma}$}

Line profiles are presented in Fig. 4 for the helium-like $\operatorname{Mg} n=4$ to $n=1$ transitions used to diagnose plasma conditions in recent opacity experiments $[6,36]$. These calculations again use standard broadening theory with the APEX low-frequency microfield component for charged radiators [37,38]. The calculations include dipole allowed couplings between the $n=4$ and 5 levels; the latter were found to impact the line profile. The calculations include Doppler and instrumental broadening where the latter assumes a Gaussian to simulate the spectral resolving power, / $\approx 1000$, in the experiments [36,39]. The figure shows the calculation with $G_{O H}$ (used in the opacity experiments $[6,36]$ ) broader than with $G_{L}$ at the same plasma conditions. In 
order to match the line wing (often used for density determination rather than the more uncertain line core), the density in the calculation with $G_{L}$ has to be increased by $\sim 25 \%$ as shown in Fig. 4 .

\subsection{Isolated lines}

Only hydrogenic or helium-like systems were considered in the comparisons above. It is known, however, that the line widths of $\mathrm{Li}^{-}$and Be-like systems display a disturbing disagreement between theoretical and experimental results [13]. These isolated lines are produced by ions with one or two electrons outside a closed $K$-shell. It is then interesting to compare the line widths using $G_{L}$ and $G_{O H}$ for these lines.

The examples in Table 3 are for the $3 \mathrm{~s}-3 \mathrm{p}$ transitions in $\mathrm{N} \mathrm{V}$ and Ne VIII. The widths ${ }_{L}$ and ${ }_{O H}$ computed with $G_{L}$ and $G_{O H}$, respectively, are compared with experimental values expt, which have been scaled to $n_{e}=10^{18} \mathrm{~cm}^{3}$ [13]. The table shows good agreement between both ${ }_{O H}$ and ${ }_{L}$ with the experimental values for $\mathrm{N} \mathrm{V}$ where other electron width models also agree with measurements [13]. On the other hand, both models underestimate expt for the Ne VIII lines by more than a factor of 2 . This disagreement for Ne VIII, however, is not unique to these two models and most calculations, including more rigorous close-coupling methods, also underestimate the width by $\sim 50 \%$ [13]. In Table 3 are also displayed calculations with $G_{L}$ neglecting the strong collisions, denoted by ${ }_{L}(=0)$. These results indicate that in these cases the strong collision correction adds significantly to the line widths.

\section{Conclusion}

In spectral line Stark-broadening theory the electron width is often computed with a secondorder term in the electron-radiator interaction providing rapid algorithms to generate line profiles. The O'Brien-Hooper model [8], which made contact with inverse bremsstrahlung, was shown to produce larger electron widths for the hydrogen Balmer series compared with those from Lee [2] where the discrepancy increases with principal quantum number of the radiating atomic levels.

Contrary to other second-order electron width models [2,3,9], the O'Brien-Hooper formalism is independent of the radiator internal states. That is, there is no explicit cutoff to avoid strong collisions where the perturbation expansion becomes invalid. It was shown that introducing a 
minimum impact parameter in the partial wave sum for the bremsstrahlung Gaunt factor significantly improves agreement with the hydrogen Balmer series results from Lee.

The inferences regarding the O'Brien-Hooper formalism rely on the accuracy of the results from Lee. An electron width formula similar to $G_{L}$, however, was found inaccurate for the $H_{13}$ line at high densities due to simultaneous strong collisions [18]. Nevertheless, calculations with $G_{L}$ yield good agreement with the Balmer series measurements [10]. Therefore inspection of Figs. 1 and 2 indicate that the O'Brien-Hooper formalism overestimates the width of all the lines in these figures.

Although the O'Brien-Hooper model compares well with experiments for the He II Ly $\alpha$ line, the Lyman series displays increasing discrepancies between the O'Brien-Hooper and Lee formulations with increasing principal quantum number. Potential errors in plasma characterization relying on these electron width models for the Mg Hey $\square \square \square \square$ were considered for recent opacity experiments [6].

The two electron width models were tested with isolated 3s-3p transitions of Li-like systems [13]. Both yielded reasonable agreement with other models including more rigorous but computationally intensive close-coupling methods. The agreement is welcome since the fast electron width models are applied to multi-electron ions [3,4]. In these cases the O'Brien-Hooper formula produced somewhat smaller line widths, which are not readily explained by simply adding the strong collision correction in the Lee model.

The behavior of the O'Brien-Hooper formalism for levels with high principal quantum number is not surprising. It was previously noted [8] that the missing strong collision cutoff made uncertain the connection between electron widths and bremsstrahlung. It was argued [8] that for charged radiators close collisions were partially neglected by excluding the bound states in the Coulomb basis set. For neutral radiators, however, there are no bound levels to exclude. Thus, the approximation appears reasonable for lines involving deeply bound levels of charged radiators but weakly bound levels are more likely to experience strong collisions and the cutoff plays a significant role. On the other hand, even for deeply bound states of hydrogen the O'Brien-Hooper formalism fails near line center and becomes worst in the line wings.

Finally, the absence or approximate treatment of strong collisions in second-order models remains one of several open questions in Stark-broadening theory. The uncertainty in these 
practical as well as the more rigorous electron width models [13] requires future high precision measurements of line profiles [40,41].

Acknowledgements: Thanks are due to R.W. Lee and R.C. Mancini for reading the manuscript and to T. Nagayama for bringing attention to this problem during his analysis of the opacity experiments. The anonymous referees are also thanked for their valuable suggestions. This work performed under the auspices of the U.S. Department of Energy by Lawrence Livermore National Laboratory under Contract DE-AC52-07NA27344. 


\section{References}

[1] H.R. Griem, Spectral Line Broadening in Plasmas (Academic Press, New York, London, 1974)

[2] R.W. Lee, JQSRT 40, 561(1988)

[3] A. Calisti et al., Phys.Rev. A42, 5433(1990)

[4] R.C. Mancini et al., CPC 63, 314(1991)

[5] D.J. Hoarty et al., Phys.Rev. Lett. 110, 265003(2013)

[6] J.E. Bailey, Nature 517, 57(2015)

[7] R.C. Mancini et al., HEDP 9, 731(2013)

[8] J.T. O’Brien \& C.F. Hooper, JQSRT 14, 479(1974)

[9] H,R. Griem, M. Blaha \& P.C. Kepple, Phys.Rev. A19, 2421(1979)

[10] W.L. Weise, D.E. Kelleher, D.R. Paquette, Phys.Rev. A6, 1132(1972)

[11] R.J. Tighe \& C.F. Hooper, Phys.Rev. A14, 1514(1976)

[12] L.A. Woltz \& C.F. Hooper, Phys.Rev. A9, 4766(1988)

[13] Yu.V. Ralchenko, H.R. Griem \& I. Bray, JQSRT 81, 371(2003), and references therein

[14] J.W. Dufty, Phys.Rev. 187,305(1969

[15] I.I. Sobelman, L.A. Vainshtein \& E.A. Yukov, Excitation of Atoms and Broadening of Spectral Lines, (Springer-Verlag, Berlin, Heidelberg, New York, 1981)

[16] C.A. Iglesias, HEDP 6, 318(2010), see Appendix C

[17] M. Abramowitz \& I.A. Stegun, Handbook of Mathematical Functions, NBS, 1972

[18] S. Ferri et al., Phys.Rev. E58, R6943(1998)

[19] R.W. Lee \& J.T. Larsen, JQSRT 56, 535(1996)

[20] H.-K. Chung et al., HEDP 1, 3(2005)

[21] C.A. Iglesias \& F.J. Rogers, Ap.J.Suppl. 79, 507(1992)

[22]] I.P. Grant, MNRAS 118, 241(1958)

[23] W.J. Karzas \& R. Latter, Ap.J. Suppl. 6, 167(1961)

[24] R.R. Johnson, JQSRT 7, 815(1967)

[25] J. Greene, Ap.J. 130, 693(1959)

[26] E.A. Smith, Phys.Rev. 166, 102(1968)

[27] T.W. Hussey, J.W. Dufty \& C.F. Hooper, Phys.Rev. A16, 1248(1977) 
[28] Alternatively, a screened potential can be introduced, however, this requires explicit calculations for different plasma conditions rather than the rapid computation by accessing the available tabular results for the pure Coulomb potential [23].

[29] The calculation of the hydrogen Balmer spectrum in Ref. 21 assumed local thermal equilibrium to obtain level populations in $\mathrm{H}_{\text {and }} \mathrm{H}^{-}$. It then computed the photon absorption and used the Kirchhoff-Planck law to compute the emission.

[30] A. Alastuey et al., Phys.Rev. A30, 2537(1984) (see Appendix A)

[31] C.A. Iglesias \& V. Sonnad, HEDP 6, 399(2010)

[32] D.R. Inglis \& E. Teller, Ap.J. 90, 439(1939)

[33] P.C. Kepple, Phys.Rev. A6, 1(1972)

[34] C.C. Smith \& D.D. Burgess, J.Phys. B11, 2087(1978)

[35] R.L. Greene \& J. Cooper, JQSRT 15, 1045(1975)

[36] T. Nagayama et al., Phys. Plasmas 21, 056502(2014)

[37] C.A. Iglesias et al., Phys.Rev. A31, 1698(1985)

[38] C.A. Iglesias et al., JQSRT 65, 303(2000)

[39] G. Loisel et al., Rev.Sci.Instrum. 83, 10E133(2012)

[40] R.E. Falcon et al., Ap.J. 806, 214(2015)

[41] M.H. Montgomery et al., HEDP; in press 
Table 1

Ratio of electron widths at $\quad={ }_{p}$ and $T=1 \mathrm{eV}$ for the $\mathrm{H}$ Balmer series

\begin{tabular}{|c|c|c|c|c|c|}
\hline & \multicolumn{2}{|c|}{$n_{e}=10^{16} \mathrm{~cm}^{3}$} & \multicolumn{2}{c|}{$n_{e}=10^{17} \mathrm{~cm}^{3}$} & \\
\hline$n$ & $G_{O H}^{\text {Born }} / G_{L}$ & $\tilde{G}_{O H}^{\text {Born }} / G_{L}$ & $G_{O H}^{\text {Born }} / G_{L}$ & $\tilde{G}_{O H}^{\text {Born }} / G_{L}$ & $\ell_{\text {min }}$ \\
\hline 3 & 1.16 & 0.95 & 1.20 & 0.94 & 3 \\
\hline 4 & 1.35 & 0.96 & 1.48 & 0.94 & 6 \\
\hline 5 & 1.55 & 0.96 & 1.77 & 0.95 & 10 \\
\hline 6 & 1.80 & 1.00 & 2.18 & 1.00 & 14 \\
\hline 7 & 1.97 & 0.99 & 2.50 & 0.97 & 19 \\
\hline 8 & 2.14 & 0.98 & 2.84 & 0.95 & 26 \\
\hline 9 & 2.33 & 0.97 & 3.23 & 0.92 & 32 \\
\hline 10 & 2.52 & 0.96 & 3.67 & 0.90 & 40 \\
\hline 11 & 2.72 & 0.95 & 4.15 & 0.87 & 49 \\
\hline 12 & 2.93 & 0.94 & 4.70 & 0.84 & 58 \\
\hline 13 & 3.16 & 0.93 & 5.29 & 0.81 & 68 \\
\hline 14 & 3.40 & 0.92 & 5.94 & 0.78 & 79 \\
\hline 15 & 3.65 & 0.91 & 6.63 & 0.74 & 91 \\
\hline 16 & 3.92 & 0.90 & 7.34 & 0.69 & 104 \\
\hline 17 & 4.21 & 0.88 & 8.06 & 0.64 & 117 \\
\hline 18 & 4.52 & 0.87 & 8.76 & 0.58 & 131 \\
\hline 19 & 4.85 & 0.86 & 9.43 & 0.53 & 146 \\
\hline 20 & 5.20 & 0.84 & 10.0 & 0.47 & 162 \\
\hline
\end{tabular}


Table 2

Ratio $G_{O H} / G_{L}$ at $={ }_{p}$ and $T=4 \mathrm{eV}$ for the He II Lyman series

\begin{tabular}{|c|c|c|c|}
\hline$n$ & $n_{e}=10^{17} \mathrm{~cm}^{3}$ & $n_{e}=310^{17} \mathrm{~cm}^{3}$ & $n_{e}=10^{18} \mathrm{~cm}^{3}$ \\
\hline 2 & 0.76 & 0.75 & 0.75 \\
\hline 3 & 0.93 & 0.94 & 0.96 \\
\hline 4 & 1.09 & 1.11 & 1.17 \\
\hline 5 & 1.24 & 1.29 & 1.38 \\
\hline 6 & 1.42 & 1.52 & 1.68 \\
\hline 7 & 1.54 & 1.67 & 1.89 \\
\hline 8 & 1.67 & 1.84 & 2.14 \\
\hline 9 & 1.80 & 2.02 & 2.39 \\
\hline 10 & 1.94 & 2.20 & 2.69 \\
\hline 11 & 2.08 & 2.40 & 3.01 \\
\hline 12 & 2.23 & 2.62 & 3.36 \\
\hline 13 & 2.38 & 2.84 & 3.75 \\
\hline 14 & 2.54 & 3.10 & 4.18 \\
\hline 15 & 2.72 & 3.37 & 4.64 \\
\hline 16 & 2.90 & 3.65 & 5.14 \\
\hline 17 & 3.09 & 3.97 & 5.66 \\
\hline 18 & 3.30 & 4.30 & 6.19 \\
\hline 19 & 3.52 & 4.66 & 6.73 \\
\hline 20 & 3.74 & 5.04 & 7.26 \\
\hline
\end{tabular}


Table 3

Comparison of electron line widths for the $3 s-3 p$ transitions scaled to $n_{e}=10^{18} \mathrm{~cm}^{3}$

\begin{tabular}{|c|c|c|c|}
\hline \multicolumn{4}{|l|}{ N V } \\
\hline$T[\mathrm{eV}]$ & $\mathrm{OH} /$ expt & ${ }_{L} /$ expt & ${ }_{L}(=0) /{ }_{\text {expt }}$ \\
\hline 6.8 & 0.86 & 0.92 & 0.55 \\
\hline 14.8 & 0.85 & 0.92 & 0.59 \\
\hline 18 & 0.87 & 0.94 & 0.62 \\
\hline 22 & 0.84 & 0.89 & 0.60 \\
\hline 24 & 0.85 & 0.90 & 0.56 \\
\hline \multicolumn{4}{|l|}{$\mathrm{Ne}$ VIII } \\
\hline$T[\mathrm{eV}]$ & $\mathrm{OH} / \mathrm{expt}$ & $L / \operatorname{expt}$ & ${ }_{L}(=0) /{ }_{\text {expt }}$ \\
\hline 30 & 0.38 & 0.45 & 0.31 \\
\hline 42 & 0.39 & 0.46 & 0.32 \\
\hline
\end{tabular}




\section{Figure Captions}

Fig 1 - Hydrogen Balmer emission spectra: Weise et al. [10] (dots) and OPAL with $G_{L}$ [21] (solid).

Fig 2 - Hydrogen Balmer bound-bound spectra computed with $G_{L}$ (solid) and $G_{O H}^{\text {Born }}$ (dash) for plasma temperature $T=1 \mathrm{eV}$ and two electron densities indicated in the figure. The higher density spectra were multiplied by 100 for clarity.

Fig 3 - Ratio of the O'Brien-Hooper to Lee $G$-functions as a function of detuning frequency for the $H \beta$ line at plasma conditions in Fig. 2: $G_{O H}^{\text {Born }} / G_{L}$ (heavy solid) and (heavy dash). $\tilde{G}_{O H}^{\text {Born }} / G_{L}$ (thin solid) and (thin dash). The plots terminate at the Weisskopf frequency.

Fig 4 - The $\mathrm{Mg} \mathrm{He \gamma}$ line profiles at $T=182 \mathrm{eV}$ with $G_{L}$ (short-dash) and $G_{O H}$ (solid) at $n_{e}=3.110^{22} \mathrm{~cm}^{3}$ plus profile with $G_{L}($ long-dash $)$ at $n_{e}=3.910^{22} \mathrm{~cm}^{3}$. 


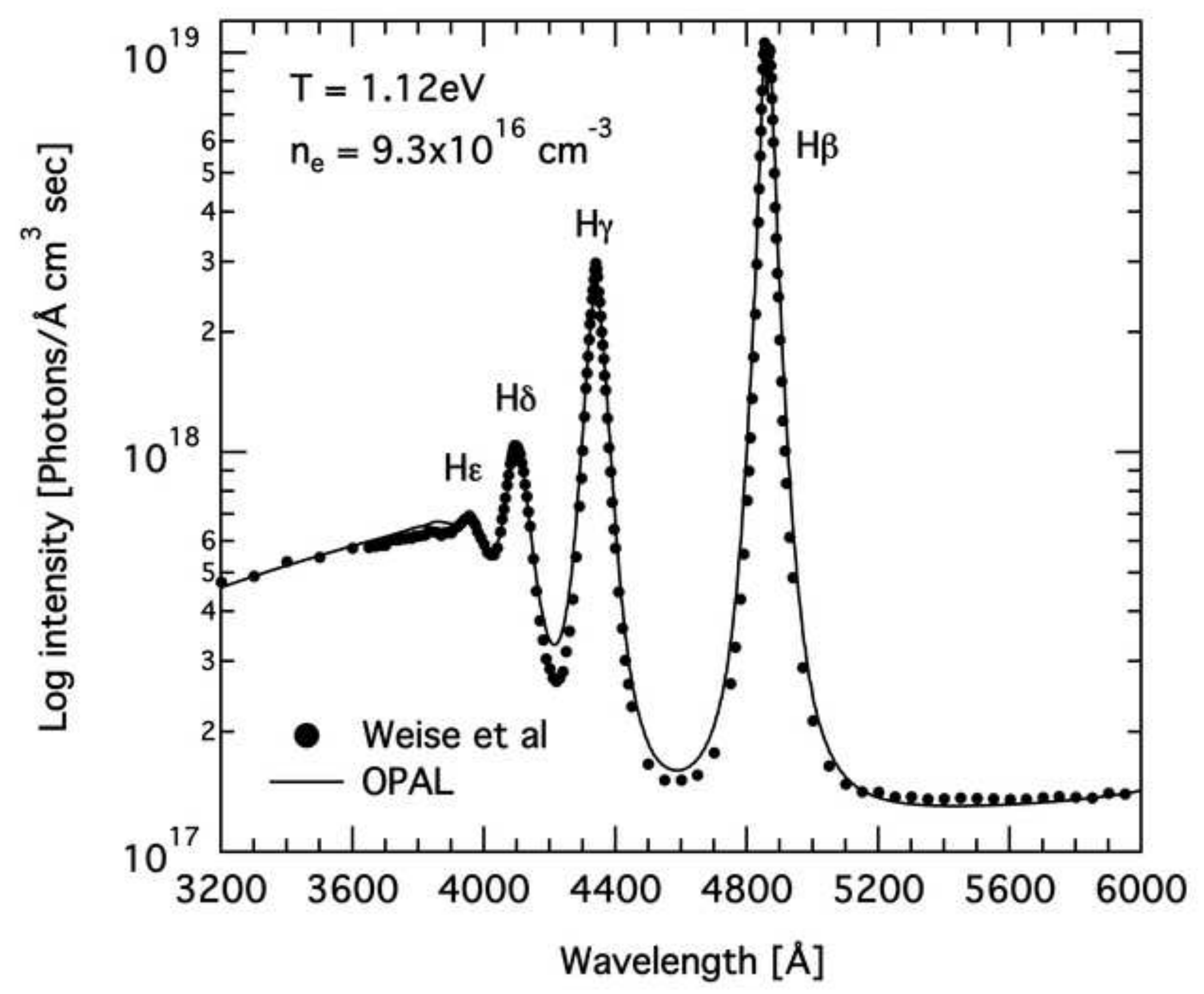




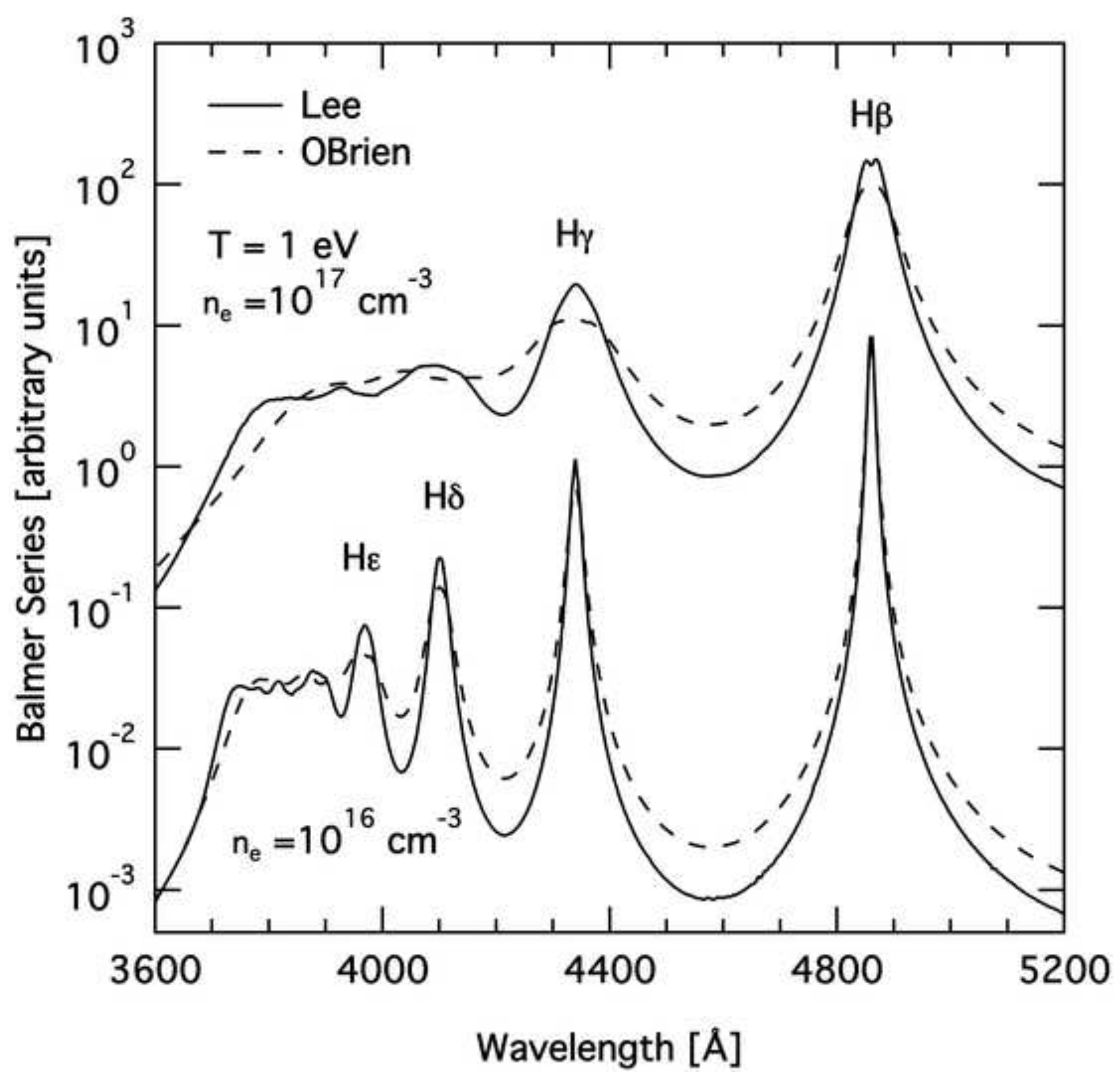




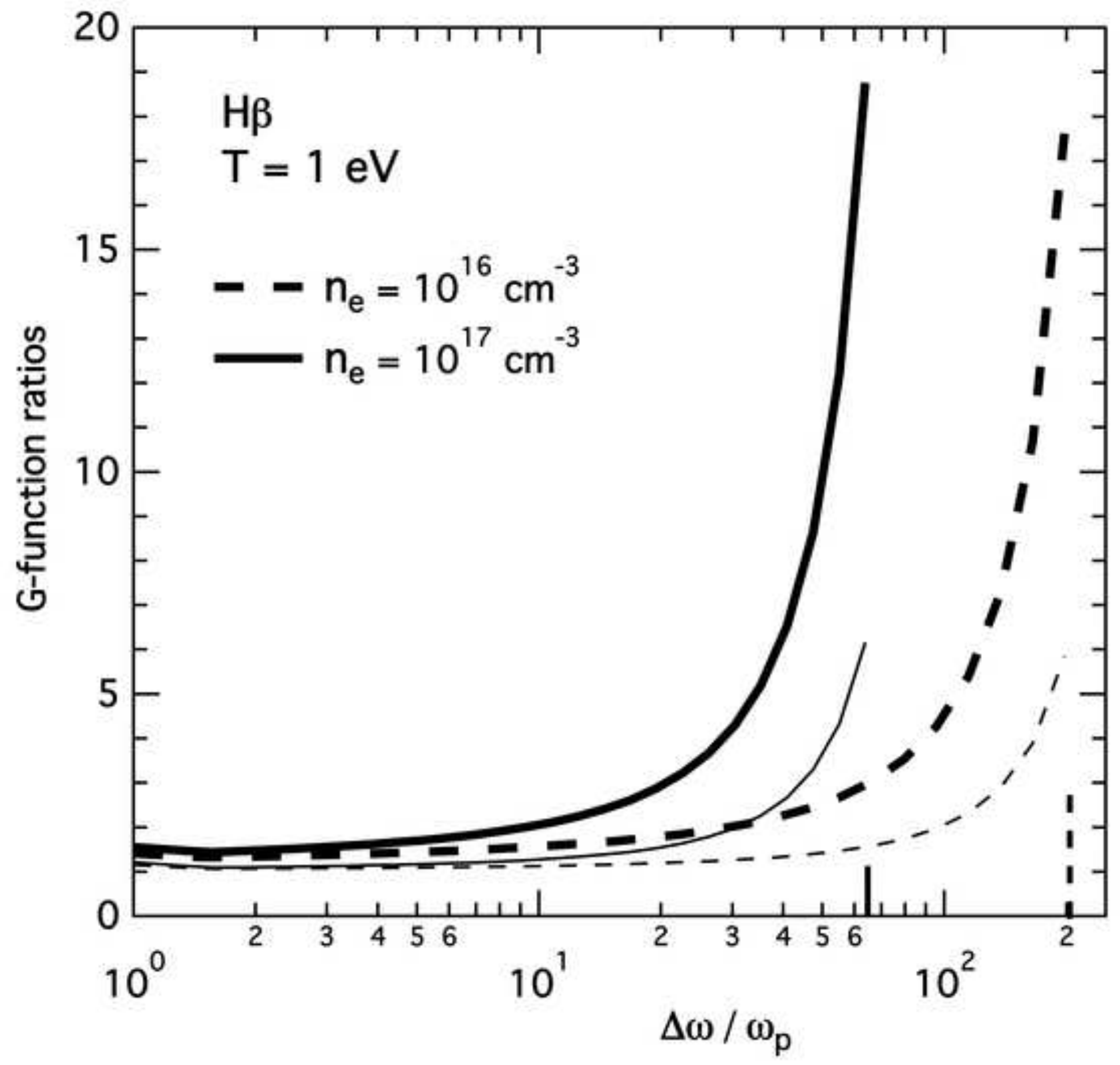




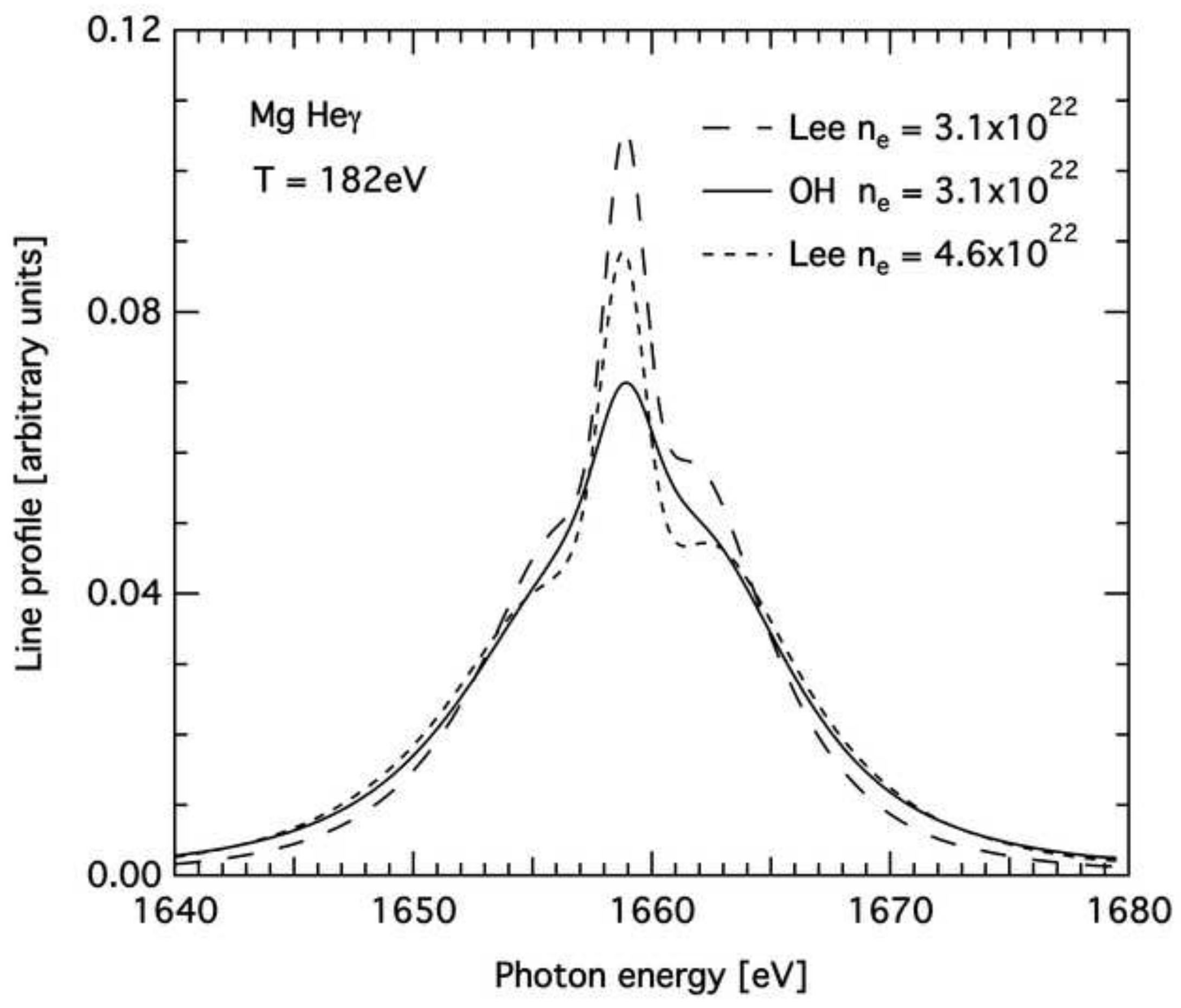

